\title{
NONLINEAR PARAMETER IDENTIFICATION OF RISK TECHNICAL SYSTEMS
}

\author{
Nikolay Petrov \\ Trakia University - Stara Zagora, Yambol, Bulgaria; \\ 8600 Yambol, Gr.Ignatiev Str. 38, nikipetrov@lycos.com
}

\begin{abstract}
This paper deals with an optimal method concerning nonlinear parameter identification of risk technical systems (automobile and railway transport, aircrafts, marine and river transport, chemical installations, munitions, information society suffering by terrorism). Unknown states of the model are built by sliding observers which converge in a finite time. Due to this property, it is possible to derive equations of the model in order to obtain an estimation law which converges to the nominal values of the parameters also in the finite time.
\end{abstract}

Keywords: Nonlinear Parameter Identification; Risk Technical Systems

\section{INTRODUCTION}

While identification in linear context it is now well established and it is widely applied, methods for nonlinear identification of risk technical systems (RTS) are nowadays uncommon. This paper deals with a new method to identify parameters of some nonlinear models. In order to identify unknown parameters, we build observes which estimate unknown states. Our approach is inspired from methods based on sliding observes. This kind of observes was introduced by Slotine, Hedrick and Misawa [1] and then used by many authors in various control problems. Our idea here is to generalize to nonlinear parameter identification the work by Utkin [2] on linear parameter identification. For instance, we will use low-pass filters in order to approximate the equivalent control and in order to avoid the chattering problems. The main advantage of our approach is its robustness and the fact that it converges in a finite time.

After describing our observer in section 2, we will apply the proposed method in section 3 in order to design a new identifier for a general class of nonlinear parameter systems $\dot{x}=f(x, \theta)$ where $x \in R^{n}$ and $\theta \in R^{p}$ is the vector of unknown parameters.

\section{DESIGN OF A SLIDING OBSERVER}

Let us consider the following class of nonlinear systems:

$$
\dot{x}_{i}=x_{i+1}, i=1, \ldots, n-1,
$$

$$
\begin{gathered}
\dot{x}_{n}=f\left(x_{1}, x_{2}, \ldots, x_{n-1}, x_{n}\right), \\
y=x_{1},
\end{gathered}
$$

where $x=\left(x_{1}, x_{2}, \ldots, x_{n}\right)^{T} \in R^{n}$ and with $f(0)=0$.

We will assume that the RTS is stable around the equilibrium point $x_{0}=0$ and that the region of attraction of $x_{0}=0$ is contained in a known ball of radius $\rho$. In the light of the observer designed by Drakunov and Utkin [3] and then studied by AhmedAli [4] and Ahmed-Ali and Lamnabhi-Lagarrigue [5], let us consider the following sliding observer:

$$
\begin{gathered}
\dot{f}_{1}=k_{1} \cdot \operatorname{sign}\left(x_{1}-f_{1}\right), \\
\dot{z}_{1}=\frac{1}{r_{1}}\left[\operatorname{sign}\left(x_{1}-f_{1}\right)-z_{1}\right], \\
\dot{\epsilon}_{i}=k_{i} \cdot \operatorname{sign}\left(k_{i-1} z_{i-1}-f_{i}\right), i=2, \ldots, n-1, \\
\dot{z}_{i}=\frac{1}{\tau_{i}}\left[\operatorname{sign}\left(k_{i-1} z_{i-1}-f_{i}\right)-z_{i}\right], \\
\dot{E}_{n}=f\left(x_{1}, k_{1} z_{1}, . ., k_{n-1} z_{n-1}\right)+k_{n}\left(k_{n-1} z_{n-1}-E_{n}\right),
\end{gathered}
$$

where $k_{i}, i=1, \ldots, n$ and $\tau_{i}, i=1, \ldots, n-1$ are suitable positive constants which will be determined later on. By defining the observation errors $e_{i}=x_{i}-E_{i}$ for 
$i=1, \ldots, n$ and the new states $\mu_{i}=x_{i}-k_{n-1} z_{n-1}$, for $i=2, \ldots, n$ we obtain equations:

$$
\begin{gathered}
\dot{e}_{i}=x_{i+1}-k_{i} \operatorname{sign}\left(x_{i}-€_{i}-\mu_{i}\right), \quad i=1, \ldots, n-1 \\
\dot{\mu}_{i}=x_{i+1}+\frac{x_{i}-\mu_{i}}{\tau_{i-1}}-\frac{k_{i-1}}{\tau_{i-1}} \operatorname{sign}\left(x_{i-1}-€_{i-1}-\mu_{i-1}\right) \\
\quad i=2, \ldots, n-1 \\
\dot{e}_{n}=f(x)-f\left(x_{1}, x_{2}-\mu_{2}, \ldots, x_{n}-\mu_{n}\right)- \\
-k_{n} \operatorname{sign}\left(x_{n}-€_{n}-\mu_{n}\right) \\
\dot{\mu}_{n}=f(x)+\frac{x_{n}-\mu_{n}}{\tau_{n-1}}-\frac{k_{n-1}}{\tau_{n-1}} \\
\cdot \operatorname{sign}\left(x_{n-1}-€_{n-1}-\mu_{n-1}\right)
\end{gathered}
$$

\section{Proposition:}

For any initial condition $x_{0} \in R^{n}$ in the region of attraction, we can find positive constants, $k_{1}, k_{2}, \ldots, k_{n}$ that are large enough and positive constants, $\tau_{1}, \tau_{2}, \ldots, \tau_{n-1}$ that are small enough such that the observation errors $e_{1}, e_{2}, \ldots, e_{n-1}$ and the states $\mu_{2}, \ldots, \mu_{n}$ converge to zero in a finite time and such that observation error $e_{n}$ converges asymptotically to zero.

\section{Proof:}

Step 1: Let us define the Lyapunov function $V_{1}=0,5 \cdot e_{1}^{2}$. Its time derivative is given by equation:

$$
\begin{aligned}
\dot{V}_{1}=e_{1} \cdot \dot{e}_{1} & =e_{1}\left[x_{2}-k_{1} \operatorname{sign}\left(e_{1}\right)\right]= \\
& =e_{1} x_{2}-k_{1} \cdot\left|e_{1}\right|
\end{aligned},
$$

therefore: $\dot{V}_{1} \leq\left|e_{1}\right| \cdot\left|x_{2}\right|_{\max }-k_{1} \cdot\left|e_{1}\right|$.

Hence, if we chose the constant $k_{1}$ such that $k_{1}>\left|x_{2}\right|_{\max }, \quad \dot{V}_{1}$ will be negative definite and a sliding mode is reached after a finite time denoted by $t_{1}$, the sliding surface being $e_{1}$. This allows us to write that equation $e_{1}(t)=\dot{e}_{1}=0, \forall t \geq t_{1}$. At this first step, we than conclude that we can find a time $t_{1}$ and a positive constant $k_{1}$ such that:

$$
\forall t \geq t_{1}, \quad\left\{\begin{array}{l}
€_{1}=x_{1}, \\
x_{2}=k_{1} \operatorname{sign}\left(e_{1}\right) .
\end{array}\right.
$$

Step 2: Let us first consider the Lyapunov function $W_{2}=0,5 \cdot \mu_{2}^{2}$. Its time derivate is given by equation:

$$
\begin{aligned}
& \dot{W}_{2}=\mu_{2} . \dot{\mu}_{2}=\mu_{2} \cdot\left[x_{3}-\frac{k_{1}}{\tau_{1}} .\right. \\
& \left.. \operatorname{sign}\left(x_{1}-\epsilon_{1}\right)+\frac{x_{2}-\mu_{2}}{\tau_{1}}\right] .
\end{aligned}
$$

Using (14) leads to, for all $t \geq t_{1}$, $\dot{W}_{2}=\mu_{2} \cdot\left(x_{3}-\mu_{2} / \tau_{1}\right)$. This implies that if we choose the positive constant $\tau_{1}$ such that equation:

$$
\dot{W}_{2} \geq\left|\mu_{2}\right| \cdot\left|x_{3}\right|_{\max }-\left|\mu_{2}\right|^{2} / \tau_{1}<0,
$$

that is, if $\tau_{1} \leq\left|\mu_{2}\right| /\left|x_{3}\right|_{\max }$.

Then there exists a finite time $t_{2}^{\prime} \geq t_{1}$ such that a sliding mode occurs on:

$$
\mu_{2}=0
$$

Let us now consider the full state Lyapunov function:

$$
V_{2}=V_{1}+0,5 e_{2}^{2}+0,5 \mu_{2}^{2}
$$

It follows that:

$$
\dot{V}_{2}=\dot{V}_{1}+e_{2} \dot{e}_{2}+\mu_{2} \dot{\mu}_{2} .
$$

Now, by using (14) and (17), we obtain:

$$
\dot{V}_{2} \leq\left|e_{2}\right| \cdot\left|x_{3}\right|_{\max }-k_{2} \cdot\left|e_{2}\right| \text {. }
$$

Hence, if we choose the constant $k_{2}$ such that $k_{2}>\left|x_{3}\right|_{\max }, \quad \dot{V}_{2}$ will be negative definite and a sliding mode is reached after a finite time denoted $t_{2}$, the sliding surface being $e_{2}=0$. This allows us to write that $e_{2}(t)=\dot{e}_{2}=0, \forall t \geq t_{2}$, at this second step, we then conclude that we can find a time $t_{2} \geq t_{2}^{\prime} \geq t_{1} \geq 0$ and positive constant $\tau_{1}$ and $k_{2}$ such that:

$$
\forall t \geq t_{1} \quad\left\{\begin{array}{l}
\xi_{2}=x_{2}, \\
x_{3}=k_{2} \cdot \operatorname{sign}\left(e_{2}\right) .
\end{array}\right.
$$

Step $n$ : This step is almost the same as the previous one. Let us first consider: 


$$
W_{n}=\frac{1}{2} \sum_{i=2}^{i=n} \mu_{i}^{2}
$$

It can easily be shown that we can find a time $t_{n}^{\prime} \geq t_{n-1}$ such that a sliding mode occurs on equation:

$$
\mu_{n}=0 \text {, }
$$

provided that the positive constant $\tau_{n-1}$ is chosen such that $\tau_{n-1} \leq\left|\mu_{n}\right| /|f(x)|_{\max }$.

The end of the proof is shown by considering the full state Lyapunov function:

$$
V_{n}=\frac{1}{2} \sum_{i=1}^{i=n} e_{i}^{2}+\frac{1}{2} \sum_{i=2}^{i=n} \mu_{i}^{2}
$$

First, we note that, for all $t \geq t_{n-1}$ :

$$
\dot{V}_{n}=e_{n} \dot{e}_{n}+\mu_{n} \dot{\mu}_{n},
$$

and hence by using (23), it follows that, for all $t \geq t_{n}^{\prime} \geq t_{n-1}$ is done $\dot{V}_{n} \leq-k_{n} \cdot e_{n}^{2}$. We then conclude that the observation error $e_{n}$ converges asymptotical to zero.

Remark: The important property of our sliding observer is that it converges in finite time expert for the last observation error. The convergence in a finite time (see Slotine et Li [6]) can be quickly explained as follows. Let us define a time varying surface $S(t)$ of the form:

$$
s(x, t)=\left(\frac{d}{d t}+\lambda\right)^{n-1} \cdot\left(x-x_{t}\right),
$$

where $x_{t}$ is the trajectory. The sliding condition is reminding a Lyapunov function $0,5 .\left(d s^{2} / d t\right) \leq-\eta \cdot|s| . \quad$ This implies that $s \leq-\eta$ if $s$ is positive. Let $t_{1}$ be the time required to hit the surface $s=0$. By integrating the equation between $t=t_{0}=0 \quad$ and $\quad t=t_{1} \quad$ leads to $s\left(t_{1}\right)-s\left(t_{0}\right) \leq-\eta \cdot\left(t_{1}-t_{0}\right)$. However, $s\left(t_{1}\right)=0$ and $t_{0}=0$. Therefore we have $t_{1} \leq s\left(t_{0}\right) / \eta$, which illustrates the convergence to the surface in a finite time.

\section{DESIGN OF A SLIDING IDENTIFIER}

Let us consider the general class of nonlinear systems $\dot{x}=f(x, \theta)$, where $x \in \square^{n}$ represents the vector of states and $\theta \in \square^{p}$ is the vector of unknown parameters. In the following we will assume that the system is identifiable and that the function $f$ is sufficiently derivable. This means that there exist two functions $\Theta$ and $\Phi$ such that:

$$
\begin{aligned}
& \theta=\Theta\left(x, \dot{x}, \ldots, x^{(q)}\right), \\
& x^{(q+1)}=\Phi\left(x, \dot{x}, \ldots, x^{(q)}\right),
\end{aligned}
$$

where $q$ is a positive integer. Therefore, the identification problem can be stated as the search for an observer converging in finite time of the following system:

$$
\mid \begin{aligned}
& \frac{d \xi_{1}}{d t}=\xi_{2}, \\
& \frac{d \xi_{2}}{d t}=\xi_{3}, \\
& \frac{d \xi_{q+1}}{d t}=F\left(\xi_{1}, \xi_{2}, \ldots, \xi_{q}\right) .
\end{aligned}
$$

with the true vector of parameters $\theta$ obtained by the design of on adoption law, denoted in the following by $\mathscr{E}(t)$.

By applying the design of the sliding observer of the previous section to the system (29), we know that there exist large enough gains $k_{i}, i=1, \ldots, n$ and small enough $\tau_{i}, i=1, \ldots, n-1$, such the new states $\xi_{i}, i=1, \ldots, q$ converge in a finite time to zero. We then immediately deduce that the adaptation law:

$$
\oiint(t)=\Theta\left(\xi_{1}, k_{1} \xi_{1}, \ldots, k_{q-1} \xi_{q-1}\right),
$$

converges also in finite time, to the nominal values of the unknown parameters.

In order to illustrate this result, we show how to find the nominal values for a synchronous machine using a quite simple model.

\section{SIMULATION RESULTS}

\subsection{DESIGN OF THE MODEL}

Let us consider a synchronous machine for aircrafts described by the following simple model of three state equations with three unknown parameters: 


$$
\mid \begin{aligned}
& \dot{i}_{d}=-\frac{R_{S}}{L} i_{d}+p \cdot \Omega \cdot i_{q}+\frac{1}{L} v_{d}, \\
& \dot{i_{q}}=-\frac{R_{S}}{L} i_{q}-p \cdot \Omega \cdot i_{d}-p \frac{\phi_{f}}{L} \Omega+\frac{1}{L} v_{q}, \\
& \dot{\Omega}=-\frac{f}{J} \Omega+\frac{p}{J} \phi_{f} i_{q} .
\end{aligned}
$$

where $i_{d}, i_{q}, v_{d}$ and $v_{q}$ are respectively the stator currents and the stator voltages in the Park reference. $\Omega$ is the mechanical speed. All the three states $i_{d}, i_{q}, \Omega$ are assumed to be measurable and the friction parameter $f$, the inertia $J$, and the number of poles $p$, are assumed to be known. The unknown parameters that we whish to identify are the resistor $R_{S}$, the indicants $L$ and the flux $\phi_{f}$.

Finally, the reference values used in the simulation are given by:

$$
\theta_{1}=R_{S} / L=9,5 ; \theta_{2}=1 / L=1,332 ; \theta_{3}=\phi_{f}=0,1
$$

\subsection{IDENTIFIABLY}

First of all, we need to know the identifiably of the RTS i.e., if we can identify the there parameters. Rewriting the RTS described by (31) using (32) the notation, $x_{1}=i_{d}, x_{2}=i_{d}, x_{3}=\Omega$ gives:

$$
\mid \begin{aligned}
& \dot{x}_{1}=-\theta_{1} x_{1}+p x_{3} x_{2}+\theta_{2} v_{d}, \\
& \dot{x}_{2}=-\theta_{1} x_{2}-p x_{3} x_{1}-p \theta_{3} \theta_{2} x_{3}+\theta_{2} v_{q}, \\
& \dot{x}_{3}=-\frac{f}{J} x_{3}+\frac{p}{J} \theta_{3} x_{2} .
\end{aligned}
$$

In order to prove the identifiably, let us apply a method developed by Lecourtier, LamnabhiLagarrigue and Walter [8] and based on generating power series. Let us consider the vector fields associated with equation (33),

$$
F_{0}(x, \theta)[.]=\left(-\theta_{1} x_{1}+p x_{3} x_{2}\right) \frac{\partial[\cdot]}{\partial x_{1}}+\Xi(x, \theta)
$$

where

$$
\begin{gathered}
\Xi(x, \theta)=\left(-\theta_{1} x_{2}-p x_{3} x_{1}-p \theta_{3} \theta_{2} x_{3}\right) . \\
\cdot \frac{\partial[\cdot]}{\partial x_{2}}+\Psi(x, \theta), \\
\Psi(x, \theta)=\left(\frac{p}{J} \theta_{3} x_{2}-\frac{f}{J} x_{3}\right) \frac{\partial[\cdot]}{\partial x_{3}} .
\end{gathered}
$$

Is analogy consider the vector fields with equation:

$$
\begin{gathered}
F_{1}(x, \theta)[.]=\theta_{2} \frac{\partial[.]}{\partial x_{1}}, \\
F_{2}(x, \theta)[.]=\theta_{2} \frac{\partial[\cdot]}{\partial x_{2}} .
\end{gathered}
$$

Let us now compute the following Lie derivatives $F_{1}\left(F_{0}(x, \theta)[].\right), \quad F_{2}\left(F_{0}(x, \theta)[].\right) \quad$ and $F_{1}\left(F_{0}\left(F_{0}(x, \theta)\right)[].\right)$ applied to the arbitrary chosen output $y=x_{1}+x_{3}$. That leads to equations:

$$
\begin{gathered}
F_{1}\left(F_{0}(x, \theta)[y]\right)=-\theta_{1} \theta_{2}, \\
F_{2}\left(F_{0}(0, \theta)[y]\right)=\frac{p}{J} \theta_{3} \theta_{2}, \\
F_{1}\left(F_{0}\left(F_{0}(0, \theta)\right)[y]\right)=\theta_{2} \theta_{1}^{2} .
\end{gathered}
$$

The correct identification of the RTS, is proven by showing that the nonlinear algebraic system, where $\theta^{\prime}=\left(\theta_{1}^{\prime}, \theta_{2}^{\prime}, \theta_{3}^{\prime}\right)$ is another triplet of parameters, has a unique solution $\theta=\theta^{\prime}$. It this following the system equations:

$$
\mid \begin{aligned}
& \theta_{1} \theta_{2}=\theta_{1}^{\prime} \theta_{2}^{\prime}, \\
& \theta_{3} \theta_{2}=\theta_{3}^{\prime} \theta_{2}^{\prime}, \\
& \theta_{2} \theta_{1}^{2}=\theta_{2}^{\prime}\left(\theta_{1}^{\prime}\right)^{2} .
\end{aligned}
$$

\section{CONCLUSIONS}

The conclusions obtained are summarized as follows:

1. In this paper, a new approach for the identification of parameters of risk technical systems was investigated.

2. This approach could be applied to a large class of nonlinear systems.

3. Moreover, this approach is robust towards noise compared to the classical method based on the recursive least square algorithm.

\section{REFERENCES}

[1] J.J. Slotine, J.K. Hedrik, E.A. Misava. On sliding observers for nonlinear systems, Trans. ASME, Jornaj of Dynamic Systems, Measurement and Control, 109, pp. 245-252, 1987. 
[2] Utkin V.I. Principles of identification using sliding regimes. Sov. Phis., Dokl., pp. 271-272, 1981.

[3] Drakunov S., V. Utkin. Sliding modes observers. Tutorial, IEEE Conference on Decision and Control, p.p. 3376-3378, 1995.

[4] Ahmed-Ali T. Controleurs-Observateurs adaptativs et robustes pour des systemes non lineaires incertains. These de Doctorat en Sciences, Universite de Paris-sud, 1998.

[5] Ahmed-Ali T., F. Lamnabhi-Lagarrigue. Sliding observer-controller design for uncertain triangular nonlinear systems. Technical Note IEEE Transactions on Automatic Control, 1999.

[6] Slotine J.J., Li Weiping. Applied nonlinear control. Prentice Hall, 1991.

[7] Elten D., D. Filbert. Identification of electrical parameters the speed of small three phase induction motors, Archiv Bd 12, pp. 379-383, 1990.

[8] Lecourtier Y., F. Lamnabhi-Lagarrigue, E. Walter. Volterra and generating power series approaches to identifiability testing. pp. 50-66, Pergamon Press, edited by E. Walter, 1997.
Nikolay Ivanov Petrov received M.Sc. degree from Georgi Benkovski Higher Military Institution of Air Forces, Dolna Mitropolia, Bulgaria - Specialty Communicative equipment of aircrafts. He got Ph.D.

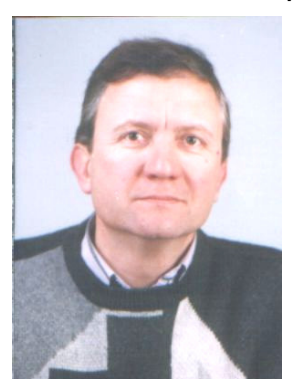
degree with doctorate thesis Optimizing and control of the technical usage of military air systems and Dr. Science degree in Automated Systems for Information Technology and Management from Military Academy - Institute for Perspective Defense Research, Sofia. Since 2001, he has been working as an Assistant Professor and Assoc. Professor at University Assen Zlatarov - Burgas, Bulgaria and Trakia University - Stara Zagora, Yambol, Bulgaria. His research activities are centered on Automated Systems, Reliability and Risk of Technical Systems and Electronic Devices for Measuring. In 2004 he graduated UNSS-Sofia, subject "Economics of the Safety". 